\title{
Review: Predictive and Prognostic Features and Their Impact on Outcome for Early-stage Breast Cancer in Mid-Western Ireland
}

\author{
K. I. Quintyne ${ }^{1,2^{\star}}$, B. Woulfe ${ }^{1,3}$, J. C. Coffey ${ }^{2,4}$ and R. K. Gupta ${ }^{1,3}$ \\ ${ }^{1}$ Mid-Western Cancer Centre (MWCC), Mid-Western Regional Hospital (MWRH), \\ Limerick, Ireland. \\ ${ }^{2}$ Graduate Entry Medical School, University of Limerick, Ireland. \\ ${ }^{3}$ Stokes Research Institute, University of Limerick, Ireland. \\ ${ }^{4}$ Department of Surgery, Mid-Western Regional Hospital (MWRH), Limerick, Ireland.
}

\section{Authors' contributions}

This work was carried out in collaboration between all authors. Author KIQ designed the study, performed the statistical analysis, and wrote the first draft of the manuscript. Authors $K I Q$ and BW managed the analyses of the study. Authors KIQ and BW managed the literature searches. All authors read and approved the final manuscript.

Review Article

Received $25^{\text {th }}$ February 2013

Accepted $21^{\text {st }}$ April 2013

Published $1^{\text {st }}$ May 2013

\section{ABSTRACT}

Aims: Predictive and prognostic features have served to allow prognostication for patients with early stage breast cancer. We sought to document our own outcomes for these features to see if our cohort corresponded to published reports.

Study Design: Retrospective pilot cohort study.

Place and Duration of Study: Department of Medical Oncology, Mid-Western Cancer Centre (MWCC), Mid-Western Regional Hospital (MWRH), Limerick, Ireland, between $1^{\text {st }}$ January 2002 and $31^{\text {st }}$ December 2002.

Methodology: A retrospective analysis was designed to include with early stage breast cancer seen at our institution for the aforementioned period, information was derived from the patients' records and indices were derived from prognostic tools. Information was analyzed using descriptive statistics and $x^{2}$ or Fisher's exact test.

Results: Seventy-seven (77) patients were found, with a median age of 52.2 years. Median overall survival of 84 months for the 10-year period of follow-up. The majority presented with moderately differentiated oestrogen receptor positive invasive ductal 
carcinoma and lymph node involvement (60\%). $64 \%$ of patients underwent mastectomy as opposed to breast conservation. Adjuvant cytotoxic chemotherapy uptake was $61 \%$, which was comparable to proportion of node positive disease. The predictive and prognostic features including axillary nodal status, tumour size, tumour grade, age at presentation and oestrogen receptor status were all significant indicators for outcome, but particularly within patients under 50 years of age.

Conclusions: This report underscores that these predictive and prognostic factors were more significant within for patients under the age of 50 years.

Keywords: Prognostic factor; predictive factor; early-stage breast cancer (EBC); survival.

\section{BACKGROUND}

Breast cancer is a common malignancy seen among European woman, with 88.4 per 100 000 diagnosed per annum [1]. Within both Western Europe and the United States (US), the mortality rates have declined in recent years possibly in part due to greater available mammographic screening programs and because of improvements in adjuvant systemic therapies for early-stage breast cancer (EBC) $[1,2]$. The Western European high mortality rates of 24.3 per 100000 , breast cancer is however still seen as a major public health concern [3].

For EBC, the ability to stratify patients into specific risk-related groups helps to determine outcome [4], and this relies on predictive and prognostic factors; and these ultimately play fundamental roles in guiding oncologists and patients and their care-givers in joint decisionmaking on potential adjuvant therapy and disease management [2,5]. Predictive factor may be defined as a clinical or biological characteristic that provides information on the likely benefit from therapy, i.e. either in terms of tumour reduction or survival $[6,7]$. Prognostic factor can be defined as a clinical or biological characteristic that is objectively measurable and provides information on likely outcome for cancer in the untreated patient $[6,7]$.

In this paper, we herein report on the predictive and prognostic factors in female patients with EBC including: axillary nodal status, tumour size, tumour histological type/grade, age at presentation and oestrogen receptor (ER) status. These features were correlated with actual outcome, with the primary objective of determining which of these clinico-pathological features was most influential within our setting, and which offered the best prognostication for patients involved. Secondary objectives of this paper were also to document incidence, menopausal profiles, and histological features of patients with EBC within our region.

\section{MATERIALS AND METHODS}

A retrospective analysis was designed to include all consecutive patients diagnosed with EBC and there were referred within the period of $1^{\text {st }}$ January 2002 to $31^{\text {st }}$ December 2002, and data was derived from the following sources:

- MWCC oncology database.

- MWRH patient charts and electronic patient records (ARIA 10.0 MR2).

- MWRH pathology reports.

- MWRH radiology reports.

- MWRH Breast Cancer Multi-Disciplinary Team (MDT) records. 
Patients included within the cohort were followed up on a regular basis for greater than 5year period on a regular basis, via a combination of clinical appointments within the MidWestern Regional Cancer Network and General Practitioners, who returned information on recurrence and survival status.

For women included in the cohort, we applied the following eligibility criteria:

- Patient age of presentation was 85 years or less.

- Have pathological data including receptor status, tumour grade, tumour grade, tumour size, number of positive lymph nodes harvested.

- Have undergone breast surgical management (including breast and axillary procedures).

- Have completed greater than 5-years of follow-up.

\subsection{Data Analysis}

The clinico-pathological features as previously described were obtained. Raw and calculated data was collated and entered into an Access $®$ database and exported into Predictive Analytic Software (PASW $尺)$. We analyzed the patient cohort by applying descriptive statistics. All results were considered significant at $p<0.05$ (two-tailed). For correlation of metric variables, Spearman rank order (rho), and for correlations of nominal variables, the $\mathrm{X}^{2}$-test, and for small sample sizes, the Fisher's exact test was used. All results of these various statistical tests are of an explorative nature. Life table survival curves were constructed using PASW version 18, for both breast cancer specific and non-specific causes.

Disease-free survival (DFS) was defined as the time from the histological diagnosis until any breast cancer event (including local or distant relapse, or death). Overall survival (OS) was calculated as the time between the histological diagnosis and the last contact date or death. Survival curves were estimated with by the Kaplan-Meier method.

\section{RESULTS}

Seventy-seven (77) female patients with invasive early stage breast cancer were diagnosed and referred to MWRH during the study period and completed 10-years of follow-up with a median overall survival of 84 months. Table 1 lists the distribution of demographic, pathologic, staging and initial treatment characteristics. The majority of the patients as would be expected were noted to have moderately differentiated oestrogen receptor positive invasive ductal carcinoma.

Fig. 1 shows the Kaplan-Meier overall survival (OS) curves based on axillary nodal status. This shows no statistical difference between the node positive and node negative groups of EBC $(p>0.05)$.

Fig. 2 shows the Kaplan-Meier OS curves based on tumour size. This shows a trend for better survival for smaller tumours, but this was not statistically significant $(p>0.05)$. 
Table 1. Summary statistics for patients in MWRH

\begin{tabular}{|c|c|c|c|c|}
\hline Variable & Number & Percentage \% & Median number & Range \\
\hline \multicolumn{5}{|l|}{ Menopausal status } \\
\hline - Premenopausal & $4 / 77$ & $5.2 \%$ & 43.6 years & 23.2-53 years \\
\hline - Postmenopausal & $35 / 77$ & $45.5 \%$ & 63.3 years & 43.1-74.1 years \\
\hline - Perimenopausal/Unknown & $38 / 77$ & $49.4 \%$ & 52.9 years & $51.9-59.5$ years \\
\hline \multicolumn{5}{|l|}{ ECOG performance status at presentation } \\
\hline - 0 & $39 / 77$ & $50.6 \%$ & & \\
\hline - $\quad 0 / 1$ & $24 / 99$ & $31.2 \%$ & & \\
\hline - 1 & $13 / 77$ & $16.9 \%$ & & \\
\hline - 2 & $1 / 77$ & $1.3 \%$ & & \\
\hline Tumour size $(\mathrm{cm})^{\mathrm{b}}$ & 77 & & $2.0 \mathrm{~cm}$ & $0.5-9.0 \mathrm{~cm}$ \\
\hline - Less than or equal $1.5 \mathrm{~cm}$ & $19 / 77$ & $24.7 \%$ & & \\
\hline - $\quad$ More than $1.5 \mathrm{~cm}$ & $57 / 77$ & $74.0 \%$ & & \\
\hline - $\quad$ Missing data & $1 / 77$ & $1.3 \%$ & & \\
\hline \multicolumn{5}{|l|}{ Tumour grade ${ }^{c}$} \\
\hline - 1 - well differentiated & $1 / 77$ & $1.3 \%$ & & \\
\hline - 2 - moderately differentiated & $61 / 77$ & $79.2 \%$ & & \\
\hline - 3 - poorly differentiated & $15 / 77$ & $19.5 \%$ & & \\
\hline \multicolumn{5}{|l|}{ Histological sub-types } \\
\hline - Infiltrating ductal carcinoma & $65 / 77$ & $84.4 \%$ & & \\
\hline - Infiltrating lobular carcinoma & $6 / 77$ & $7.8 \%$ & & \\
\hline - $\quad$ Mixed ductal and lobular carcinoma & $3 / 77$ & $3.9 \%$ & & \\
\hline - Infiltrating breast carcinoma - NOS & $3 / 77$ & $3.9 \%$ & & \\
\hline \multicolumn{5}{|l|}{ Oestrogen receptor (ER) status } \\
\hline - Negative & $25 / 77$ & $32.5 \%$ & & \\
\hline - Positive & $52 / 77$ & $67.5 \%$ & & \\
\hline - Unknown & $0 / 77$ & $0 \%$ & & \\
\hline \multicolumn{5}{|l|}{ Lymph node stage $^{d}$} \\
\hline - $\quad \mathrm{N} 0$ - no regional lymph nodes & $30 / 77$ & $39.0 \%$ & & \\
\hline - $\quad \mathrm{N} 1-$ micro-metastases or metastases in $1-3$ & $23 / 77$ & $29.9 \%$ & & \\
\hline lymph nodes & $14 / 77$ & $18.2 \%$ & & \\
\hline
\end{tabular}


- $\quad \mathrm{N} 2$ - metastases in 4 - 9 lymph nodes

\begin{tabular}{ll}
$10 / 77$ & $13.0 \%$ \\
& \\
$47 / 77$ & $61.0 \%$ \\
& \\
$20 / 77$ & $26.0 \%$ \\
$31 / 77$ & $40.3 \%$ \\
$26 / 77$ & $33.8 \%$ \\
& \\
$47 / 77$ & $61.0 \%$ \\
$19 / 77$ & $24.7 \%$ \\
$11 / 77$ & $14.3 \%$ \\
& \\
$27 / 77$ & $35.1 \%$ \\
$49 / 77$ & $63.6 \%$ \\
$1 / 77$ & $1.3 \%$ \\
\hline
\end{tabular}

- $\mathrm{N} 3$ - metastases in 10 or more lymph nodes

- Node positive

$61.0 \%$

$\mathrm{AJCC}^{\mathrm{e}}$ Stage

- Stage I

- Stage II

- Stage III

Adjuvant Chemotherapy ${ }^{\dagger}$

- Offered and undertaken

- Not offered

- Offered but not undertaken

Surgical Intervention ${ }^{\mathrm{g}}$

- Breast conserving surgery

- Mther $^{\mathrm{h}}$

\section{Abbreviations}

ECOG - Eastern Co-Operative Oncology Group.

NOS - Not otherwise specified.

a: All patients included were on Caucasian extraction.

${ }^{b}$ : Measured pathologically.

c: Assessed using the Modified Bloom and Richardson system.

: Based on Tumour node metastases staging system for carcinoma of the breast (Aebi S et al, 2011).

e: The American Joint Committee on Cancer.

: Adjuvant chemotherapy included -

- First generation regimes $-A C * 4, C M F * 6-66 \%(31 / 47)$.

- Second generation regimes - FEC(60) * 6 , FEC(100) * 6 and $A C / T(21)$ * $8-34 \%(16 / 47)$

g: All patients underwent axillary nodal clearance as sentinel lymph biopsy practice was not established within MWRH in 2002.

$h$ : One patient presented with no detectable breast primary (on conventional radiology including mammography and ultrasonography) and only axillary disease, and her only surgical intervention was axillary node clearance. 


\section{Survival Functions}

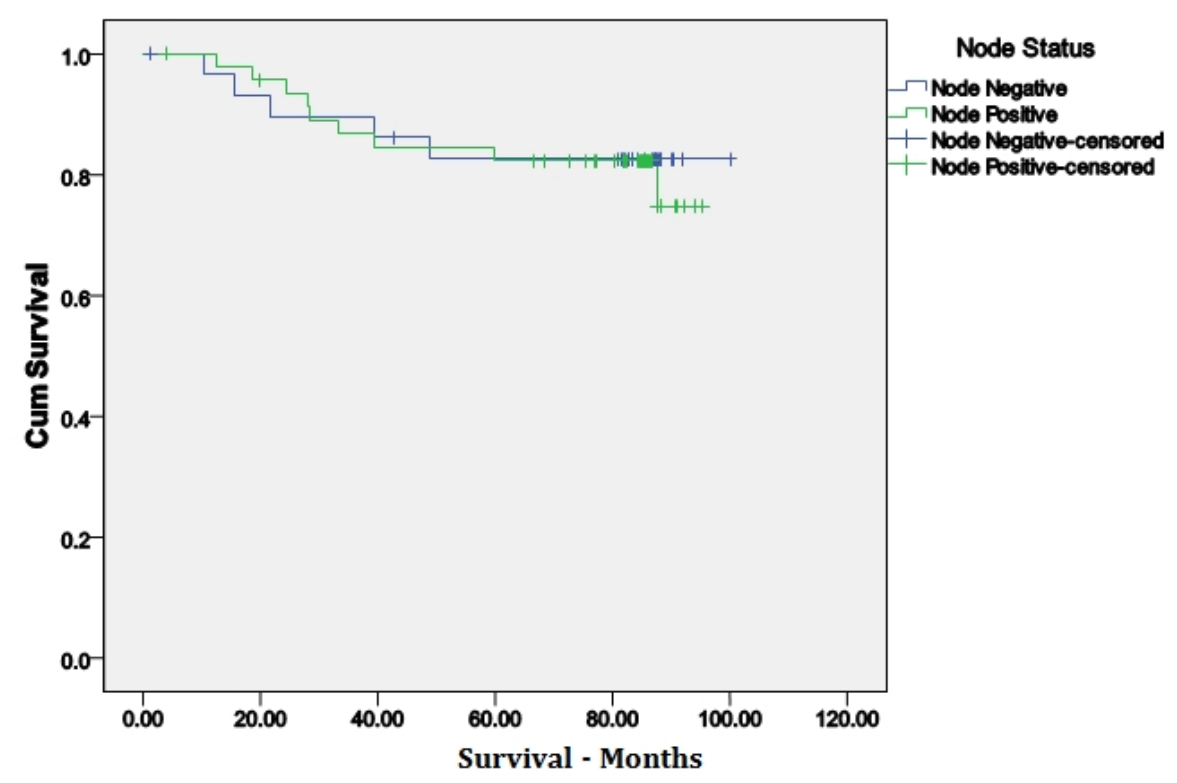

Fig. 1. Kaplan-Meier OS curves for EBC patients based on axillary nodal status

\section{Survival Functions}

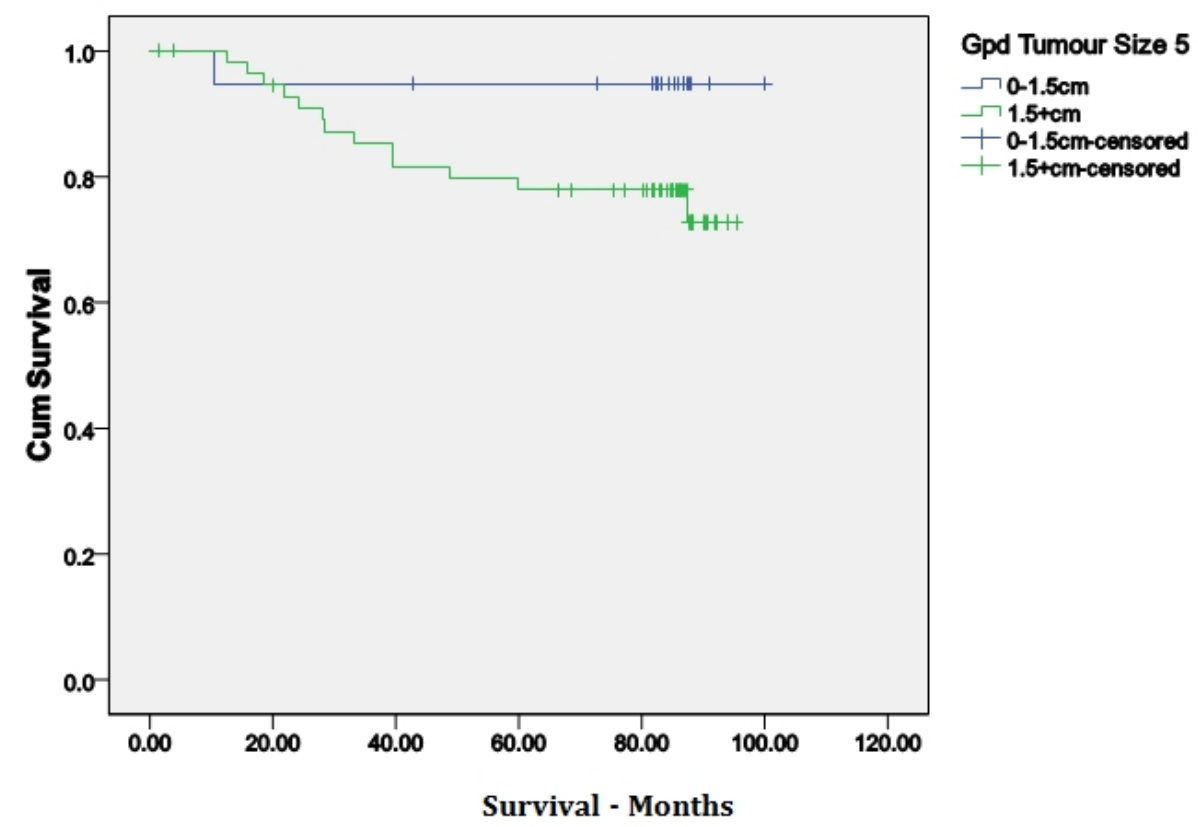

Fig. 2. Kaplan-Meier OS curves for EBC patients based on tumour size $(0.0-1.5 \mathrm{~cm}$ vs $>1.5 \mathrm{~cm}$ ) 
Fig. 3 shows the Kaplan-Meier OS curves based on tumour grade. This shows a trend for better survival for lower grade (G) tumours (i.e. G1 and G2) as opposed to G3 tumours, but this was not statistically significant $(p>0.05)$.

Fig. 4 shows the Kaplan-Meier OS curves based on age at presentation. This shows a trend for better survival for patients under the age of 50 years, but this was not statistically significant $(p>0.05)$. The age of 50 years was chosen as it offered a more even distribution of the patient cohort, it is the start age for undertaking screening mammography and menopausal data within the group was not so evenly matched as seen in Table 1.

Fig. 5 shows the Kaplan-Meier OS curves based on ER status. This shows statistical significance for survival for patients with ER positive EBC $(p<0.05)$.

Figs. 6,7 and 8 show the Kaplan-Meier OS curves based on axillary nodal status, tumour size and ER status for patients stratified by age. This revealed that for patients under 50 years of age at presentation, there was statistical significance for survival $(p<0.05)$ based on all the aforementioned criteria. This was not seen for patients over 50 years, as the ER status remained the only factor that showed statistical significance for survival in the patients over 50 years $(p<0.05)$.

\section{Survival Functions}

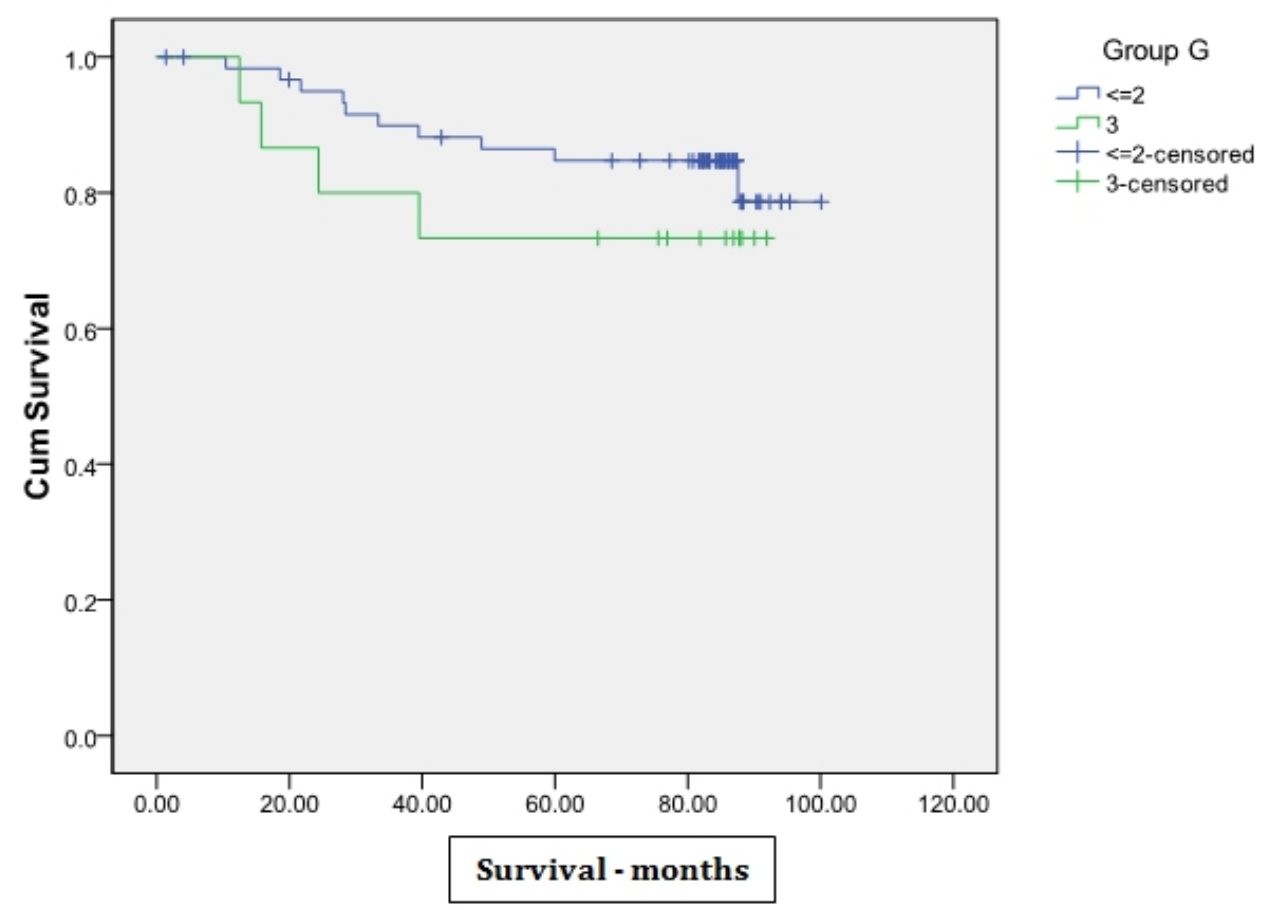

Fig. 3. Kaplan-Meier OS curves for EBC patients based on tumour grade 


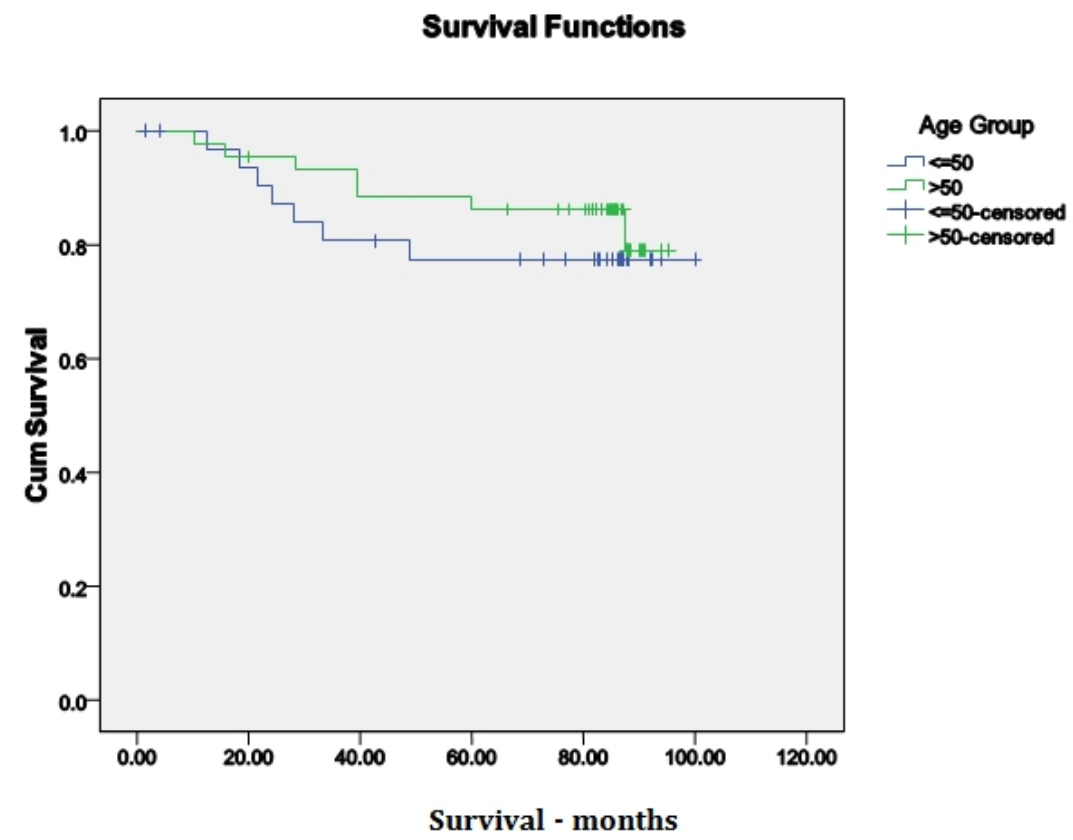

Fig. 4. Kaplan-Meier OS curves for EBC patients based on age at presentation Number of patients less than or equal 50 years $=\mathbf{3 3}$ Number of patients more than $\mathbf{5 0}$ years $=44$

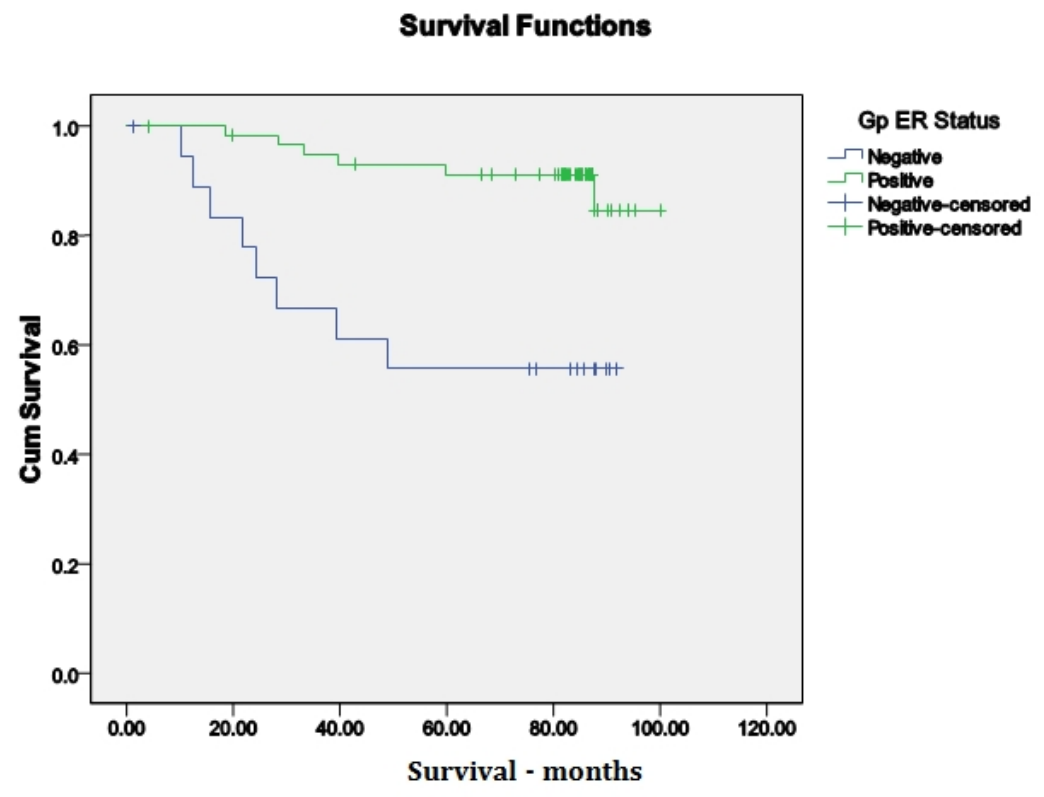

Fig. 5. Kaplan-Meier OS curves for EBC patients based on ER status (ER Positive vs. ER Negative) 


\section{Survival Functions}

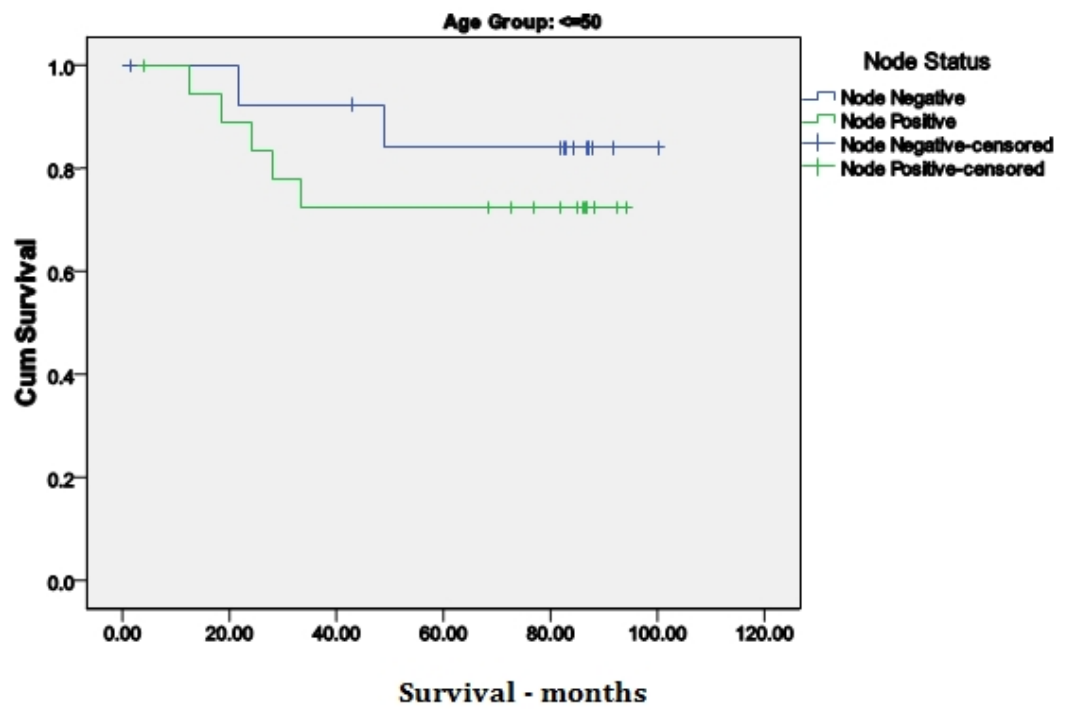

(a)

\section{Survival Functions}

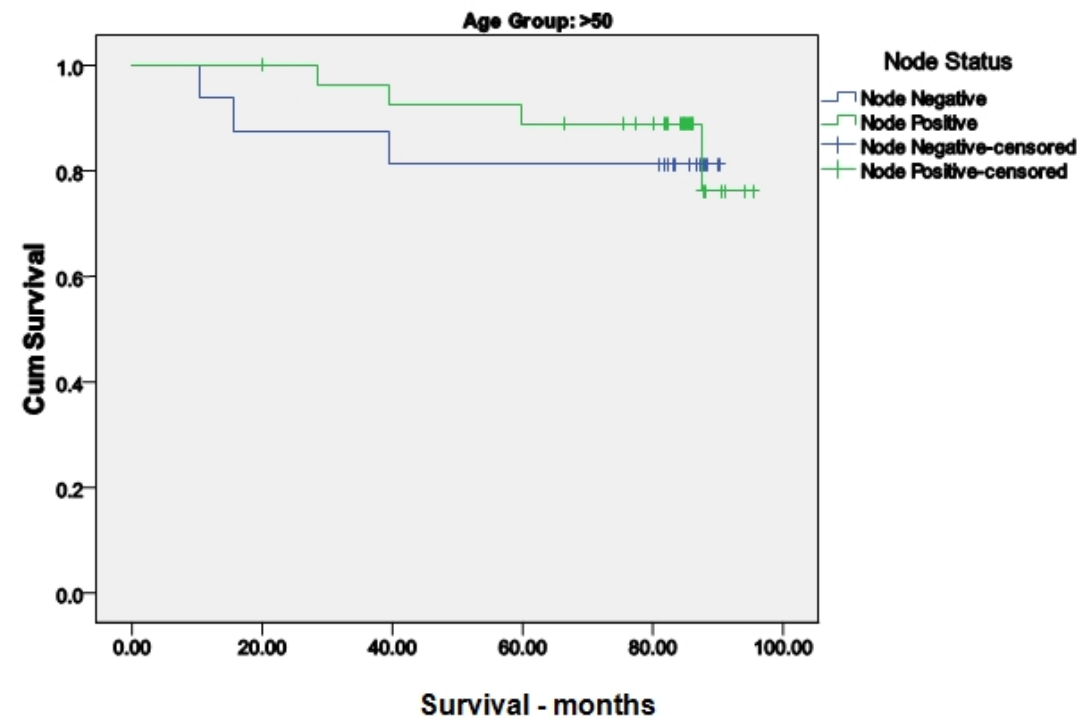

(b)

Fig. 6. Kaplan-Meier OS curves for EBC patients by axillary nodal status split by age group

(a) (Under 50 years of age: $p<0.05)$ - Number of patients $\leq 50$ years $=33$, Number of patients $\leq$ 50 years + node negative $=14$, Number of patients $\leq 50$ years + node positive $=19$.

(b) (Over 50 years of age: $p>0.05$ ) - Number of patients $>50$ years $=44$, Number of patients $>$ 50 years + node negative $=16$, Number of patients $>50$ years + node positive $=28$. 


\section{Survival Functions}

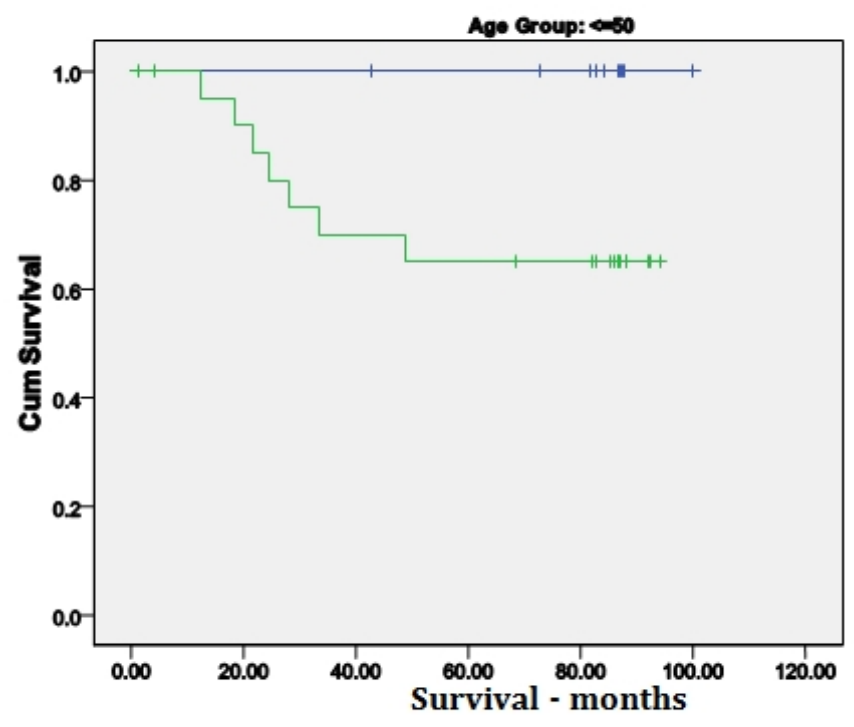

\section{Gpd Tumour Size 5}

$\neg 0-1.5 \mathrm{~cm}$

$\neg 1.5+\mathrm{cm}$

$+0-1.5 \mathrm{~cm}-\mathrm{censored}$

1.5tcm-censored

(a)

\section{Survival Functions}

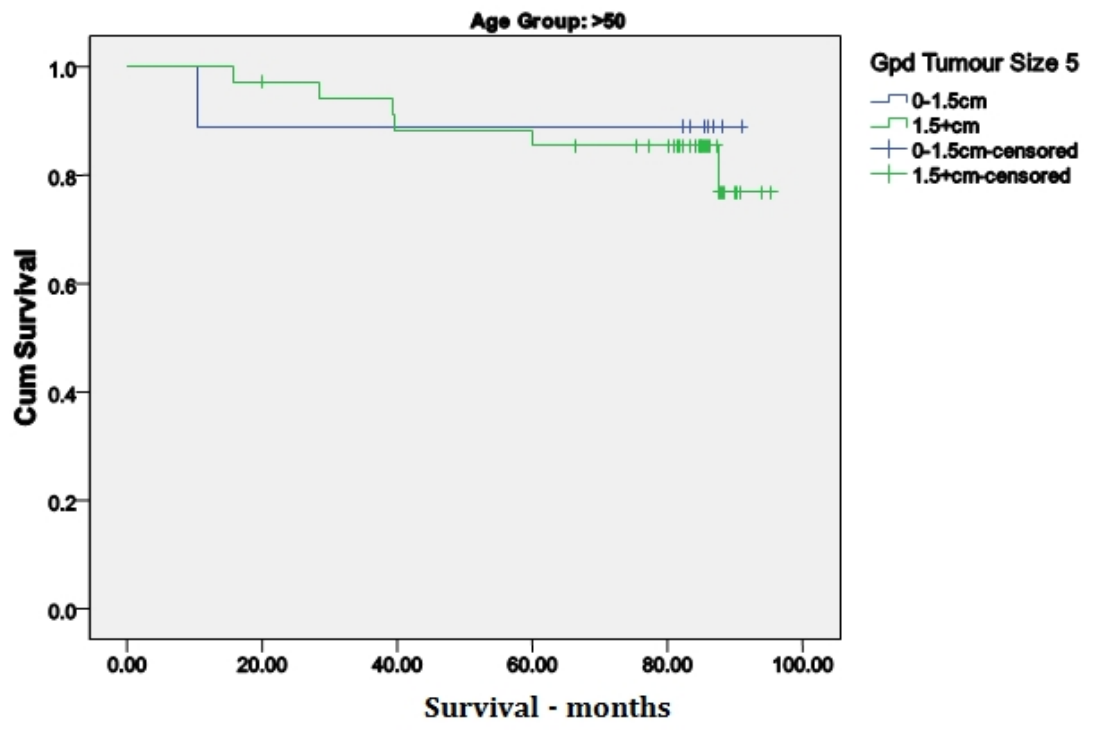

(b)

Fig. 7. Kaplan-Meier OS curves for EBC patients by tumour size split by age group

(a) (Under 50 years of age: $p<0.05$ ) - Number of patients $\leq 50$ years $=33$, Number of patients $\leq 50$ years + tumour $\leq 1.5 \mathrm{~cm}=10$, Number of patients $\leq 50$ years + tumour $>1.5 \mathrm{~cm}=22$, Missing data -1 .

(b) (Over 50 years of age: $p>0.05$ ) - Number of patients $>50$ years $=44$, Number of patients $>50$ years + tumour $\leq 1.5 \mathrm{~cm}=9$, Number of patients $>50$ years + tumour $>1.5 \mathrm{~cm}=35$. 


\section{Survival Functions}

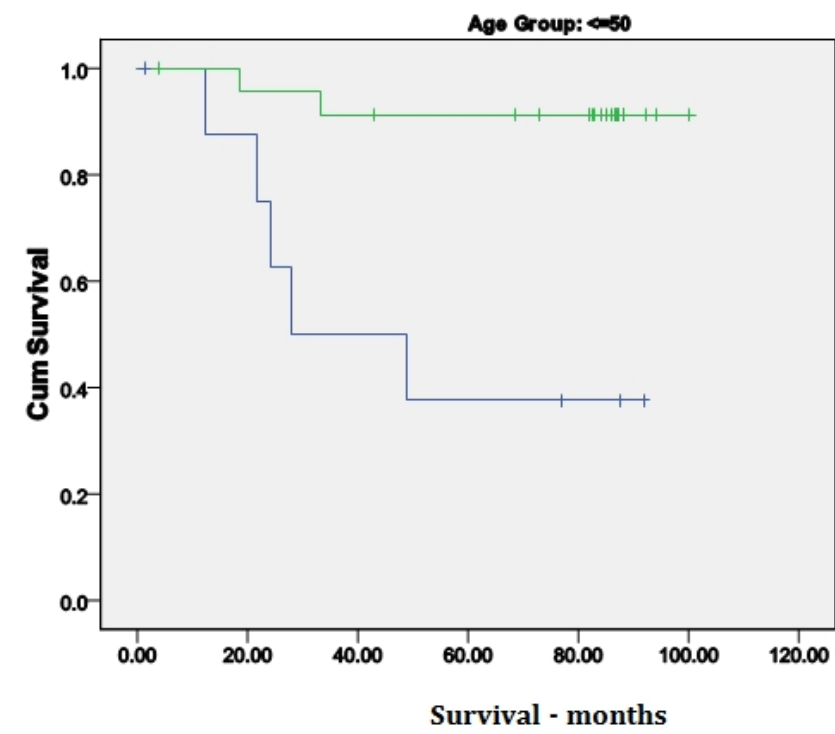

Gp ER Status

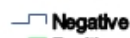

$\neg$ Positive

+ Negative-censored

+ Positive-censored

(a)

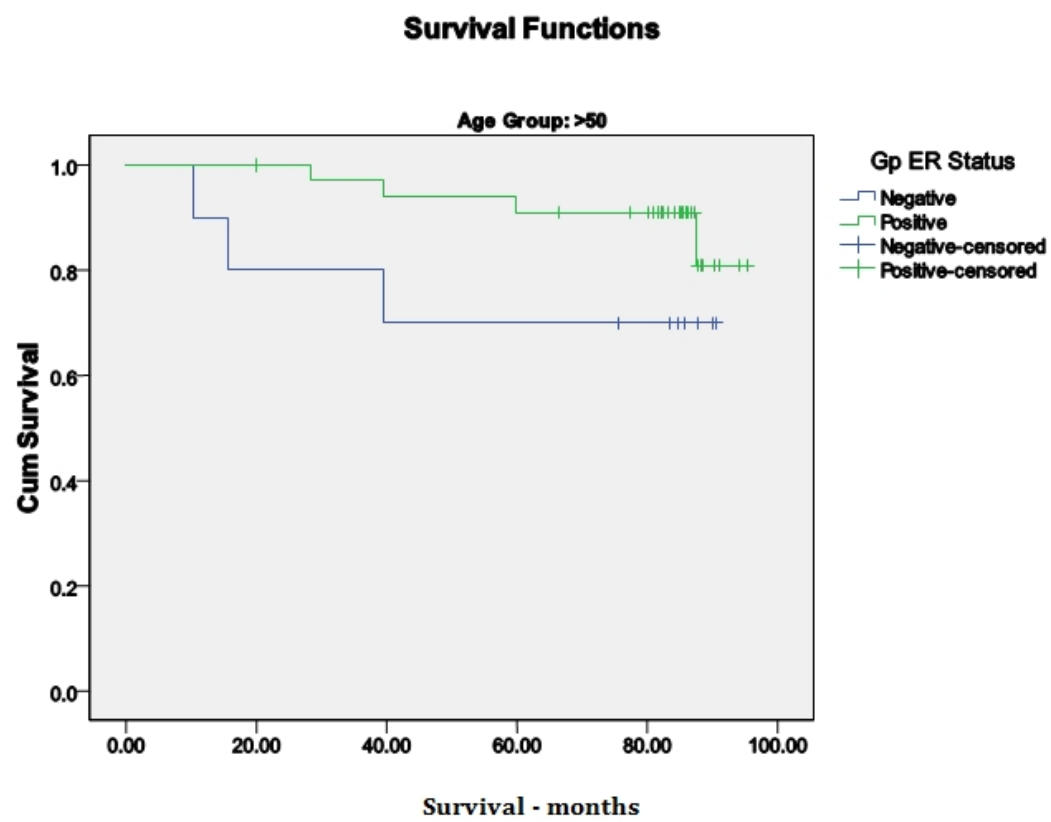

(b)

Fig. 8. Kaplan-Meier OS curves for EBC patients by ER status split by age group

(a) (Under 50 years of age: $p<0.05$ ) - Number of patients $\leq 50$ years $=33$, Number of patients $\leq 50$ years + node negative $=9$, Number of patients $\leq 50$ years + node positive $=24$.

(b) (Over 50 years of age: $p<0.05$ ) - Number of patients $>50$ years $=44$, Number of patients $>50$ years + node negative $=10$, Number of patients $>50$ years + node positive $=34$. 


\section{DISCUSSION}

Given the variety of histological sub-types and associated clinical manifestations that are seen, breast cancer can consequently be considered as a heterogeneous condition. As a result of this, the determination of an outcome for patients with EBC requires meticulous review of as many clinical and pathological parameters as possible to allow for the best prediction and prognostication.

A predictive factor in EBC may be used to facilitate the organization of an appropriate therapeutic pathway from which benefit can be derived $[6,7]$. While a prognostic factor in $\mathrm{EBC}$, may be used to determine the potential outcome without intervention $[6,7]$.

The clinical importance of employment of predictive and prognostic factors into clinical practice when assessing any new patient with EBC was highlighted by Clark, when these pivotal issues were raised: the first, was to determine patients with EBC for whom the use of adjuvant systemic therapy will not provide significant improvement in disease-free survival (DFS) or overall survival (OS) without compromise to quality of life (QOL) by incurring toxicity; the second is to identify patients with EBC for whom the use of adjuvant systemic therapy will not out-weigh the potential side-effect profile; and the third, is to choose patients with EBC, that are more or less likely to benefit from other forms of therapy [8].

The primary objective of this work was to elucidate our experience and to see whether established predictive and prognostic factors in EBC were related to outcome of patients within our region.

\subsection{Prognostic Factors}

\subsubsection{Axillary nodal status (ANS)}

Our results have shown that this parameter did not have statistically significant impact on the OS for the entire cohort; but in the under 50 years sub-group, it was seen to be significant factor for OS. The significance of this might be related to the fact that breast cancer in younger patients is a more aggressive entity, albeit with lower pathological burden from the onset. Thus this cohort may benefit from a more aggressive systemic regimen in order to ensure better outcome.

ANS is traditionally seen as the most significant prognostic factor in patients with EBC, because the involvement of axillary lymph nodes is linked directly to the potential for distant recurrence $[2,9,10]$. Historically, the lymph node status was assessed by axillary node dissection (ALND), which involves removal and collection of level I and II lymph nodes. More recently, the adoption or incorporation of sentinel lymph node biopsy (SLNB) within the surgical management of patients with lymph node negative EBC has become the mainstay $[1,11]$. Debate has arisen on what constitutes the optimal pathological assessment of sentinel lymph nodes $(\mathrm{SN})$, whether traditional haematoxylin and eosin $(\mathrm{H}+\mathrm{E})$ staining or by immunohistochemistry (IHC) is preferential, given that treatment decisions might be influenced by the outcome. Hansen et al devised a prospective model looking at this issue, and reported that there was no significant difference in DFS or OS between SN: $\mathrm{H}+\mathrm{E}$ negative + IHC negative and SN: H+E negative + IHC positive [12]. 


\subsubsection{Tumour grade}

Our results have revealed that this parameter was not statistically significant for OS, although there was a trend towards better OS with patients with Grades 1 and 2 EBC as opposed to Grade 3 EBC. It was noted however in the less than 50 years sub-group, that Grades $1+2 \mathrm{EBC}$ has statistically significant better OS. The explanation for this might be as for ANS with respect to the disparity seen in patients less than 50 years, with the trend for the overall group being related to the size of the cohort, as it lacked sufficient numbers for significance.

Tumour grade is a fundamental prognostic factor in patients with $\mathrm{EBC}$, where higher grade tumours are associated with a poorer outcome and lower OS [13,14]. Le Doussal et al. demonstrated and documented a better outcome, where 1262 were reviewed, and better 5year survival was observed [13].

\section{$\underline{\text { 4.1.3 Lymphovascular invasion (LVI) }}$}

Our review was retrospective, and this feature was not uniformly reported on pathology reports, whether this finding was present or absent, making its reporting in this setting unreliable and consequently difficult to interpret. LVI has been shown to have significance in EBC with respect to risk of local and distant recurrence [2]. This was best demonstrated in EBC where recurrence rates in stage I disease was quoted for LVI-positive as $38 \%$ as compared to LVI-negative 22\% [15], however this feature is normally employed in conjunction with other clinic-pathological parameters in the decision-making process of devising a systemic treatment strategy in patients who do not have lymph node involvement, as it lacks the strength to stand on its own [9].

\subsection{Predictive and Prognostic Factors}

\subsubsection{Oestrogen receptor (ER) status}

Our review has shown that this parameter was significant statistically with respect to its presence in the OS of patients with EBC. This finding was independent of age, unlike the other factors examined within this review.

ER positivity can be both predictive and prognostic in EBC. The predictive value of ER status positivity has been most appreciated with use of hormonal agents initially tamoxifen and more recently the aromatase inhibitor including anastrozole [1,2]. The Early Breast Cancer Trialists' Collaborative Group, examined tamoxifen for 5 years, given in the adjuvant setting following definitive breast surgery noted proportional reductions in recurrence and mortality of $47 \%$ and $26 \%$, respective in patients with ER positive EBC [16]. In postmenopausal patients with EBC the use of upfront aromatase inhibitors used for 5 years showed modest survival benefits as compared to tamoxifen [17]. The prognostic value of ER status is harder to review, as most patients with EBC with ER positivity will potentially be using either tamoxifen or aromatase inhibitor, and assessing patients not undergoing hormonal manipulation is difficult due to its rarity, so prospective or retrospective review is not often feasible [2]. 


\subsubsection{HER2/neu status}

Our review was retrospective, and this feature was not routinely evaluated and placed on pathology reports, consequently we are not able to review this in our cohort. Within published reports, it is seen within approximately $30 \%$ of patients with EBC $[1,18]$. Increased expression of this proto-oncogene have been associated with enhanced tumour aggressiveness, increased rates of recurrence and mortality $[1,19]$, hence both the predictive and prognostic nature of this parameter.

The secondary objectives with this piece were to also compare our data to findings in the published data. Our results revealed the median age of presentation for patients with early stage breast cancer was 52.2 years, and was lower than figures published for the US SEER database of 61 years [20], and UK (Caucasians only) of 67 years [16]. The significance of these differences is not clear, but the impact that a younger age at presentation (patients less 35 years at presentation) can have on the natural history of EBC is well documented with this disease being more aggressive.

Our cohort was similar to others in terms of distribution of histological sub-types were $80 \%$ of the group had ductal carcinoma; approximately $8 \%$ had lobular carcinoma with just fewer than $10 \%$ having other histology. This pattern was similar to figures published [21]. The majority of these tumours were Grades 2 and 3, which was also in keeping with standard data. For this group, approximately $67 \%$ showed oestrogen (ER) positivity and this was in keeping with conventional data [22]. $60 \%$ of the samples were also noted to have lymph node involvement, which is higher than seen figures quoted in some series of almost $35 \%$ [23].

Almost $64 \%$ of patients within the group had undergone mastectomy as their primary surgical therapy, this was not in keeping with data available, where breast conservation was the primary modality employed, with some series quoting 60-77\%. [1] Review of subsequent data within our unit is presently underway, to see if this trend has changed within more recent times, with the formal introduction of a screening program, which would be more likely to diagnose smaller primary breast lesions.

Uptake of adjuvant chemotherapy was approximately $61 \%$, but given the comparable amount of lymph node involvement for this group of 77 patients, this proportion is understandable.

\section{CONCLUSION}

This retrospective review is a first look at our own experience within the Ireland and offers good insight into our own specific clinico-pathological imprint on EBC. Within our cohort, it was noted that established prognostic and predictive factors were more statistically significant in EBC patients who were less than 50 years. We will review these features again with a larger cohort to reaffirm our preliminary findings. 


\section{CONSENT}

Not applicable.

\section{ETHICAL APPROVAL}

Not applicable.

\section{COMPETING INTERESTS}

All authors included in this piece disclose that they have no financial and personal relationships with other people or organizations that could inappropriately influence (bias) their work.

\section{REFERENCES}

1. Aebi S, Davidson T, Gruber G, et al. Primary breast cancer: ESMO clinical practise guidelines for diagnosis, treatment and follow-up. Ann Oncol. 2011;22(6):vii12-vi24. Doi:10.1093/annonc/mdr371.

2. Cianfrocca M, Goldstein LJ. Prognostic and predictive factors in early-stage breast cancer. The Oncologist. 2004;9:606-616

3. Coughlin SS, Ekwueme DU. Breast cancer as a global health concern. Cancer Epidemiol. 2009;33(5):315-318.

4. Quintyne KI, Woulfe B, Keane RM, et al. Clinico-pathological features and their influence on outcome for early-stage breast cancer in Mid-Western Ireland. http://www.asco.org/ascov2/Meetings/Abstracts?\&vmview=abst detail view\&conflD=1 00\&abstractID=60582. Accessed 29 ${ }^{\text {th }}$ March 2012 (Poster presentation 2010 Breast Cancer Symposium).

5. Moons KGM, Royston $\mathrm{P}$, Vergouwe, $\mathrm{Y}$, et al. Prognosis and prognostic research: what, why, and how? BMJ. 2009;338:1317-1320. Doi:10.1136/bmj.b375.

6. Italiano A. Prognostic or predictive? It's time to get back to definitions, J Clin Oncol. 2011;29(35):4718. Doi:10.1200/JCO.2011.38.3729.

7. Henderson IC, Patek AJ. The relationship between prognostic and predictive factors in the management of breast cancer Breast Cancer Res Treat. 1998;52(1-3):261-288. Doi.10.23/a:1006141703224

8. Henderson IC, Patek AJ. The relationship between prognostic and predictive factors in the management of breast cancer. Breast Cancer Res Treat. 1994;52(1-3):261-288. Doi.10.23/a:1006141703224.

9. Gurleyik G, Gurleyik E, Aker F, et al. Lymphovascular invasion, as a prognostic marker in patients with invasive breast cancer. Acta Chir Belg. 2007;107:284-287

10. Kim J-Y, Ryu M-R, Choi B-O, et al. The prognostic significance of the lymph node ration in the axillary lymph node positive breast cancer. J Breast Cancer. 2011;14(3):204-212

11. Albertini JJ, Lyman GH, Cox C, et al. Lymphatic mapping and sentinel node biopsy in the patient with breast cancer. JAMA. 1996;276:1818-1822.

12. Hansen NM, Grube BJ, Te W, et al. Clinical significance of axillary micrometastases in breast cancer: how small is too small? Proc Am Soc Clin Oncol. 2001;20:24a. 
13. Le Doussal V, Tubiana-Hulim M, Friedman S, et al. Prognostic value of histologic grade nuclear components of Scarff-Bloom-Richardson (SBR). An improved score modification based on a multivariate analysis of 1262 invasive ductal breast carcinomas. Cancer. 1989;64:1914-1921.

14. White J, Morrow M, Moughan J, et al. Compliance with breast conservation standards for patients with early stage breast carcinoma. Cancer. 2003;193:38-43.

15. Rosen PP, Groshen S, Saigo PE, et al. Pathological prognostic factors in stage I (T1N0M0) and stage II(T1N1M0) breast carcinoma; a study of 644 patients with median follow-up of 18 years. J Clin Oncol. 1989;7:1239-1251.

16. Early Breast Cancer Trialists' Collaborative Group. Tamoxifen for early breast cancer: an overview of the randomised trials. Lancet. 1998;351:930-942.

17. Colleoni M, Giobbie-Hurder A, Regan MM, et al. Analyses adjusting for selective crossover show improved overall survival with adjuvant letrozole compared with tamoxifen in the BIG 1-98 study. J Clin Oncol. 2011;29:1117-1124.

18. Slamon DJ, Clark GM, Wong SG, et al. Human breast cancer: correlation of relapse and survival with amplification of HER-2/neu oncogene. Science. 1987;235:177-182.

19. Winstanley J, Cooke T, Murray GD, et al. The long term prognostic significance of Cerb-2 in primary breast cancer. Br J Cancer. 1991;63:447-450.

20. Howladen N, Noone AM, Krapcho M, et al. SEER Cancer Statistics Review, 19752008, National Cancer Institute. Bethesda, MD, http://seer.cancer.gov/csr/1975 2008/. Accessed $1^{\text {st }}$ February 2012.

21. Ungat AM, Xie L, Morriss J, et al. Survival of women with breast cancer in Ottawa, Canada: variation with age, stage, histology, grade and treatment. $\mathrm{Br} \mathrm{J}$ Cancer. 2004;90(6):1138-1143. Doi:10.1038/sj.bjc.6601662.

22. Campbell HE, Gray AM, Harris AL, et al. Estimation and external validation of a new prognostic model for predicting recurrence-free survival for early breast cancer patients in the UK. Br J Cancer. 2010;103:776-786. Doi:10.1038/sj.bjc.6605863.

23. Olivotto IA, Bajdik CD, Ravdin PM et al, Population-based validation of the prognostic model ADJUVANT! for early breast cancer. J Clin Oncol. 2005;23:2716-2725. Doi:10.1200/jco.2005.06.178.

(C) 2013 Quintyne et al.; This is an Open Access article distributed under the terms of the Creative Commons Attribution License (http://creativecommons.org/licenses/by/3.0), which permits unrestricted use, distribution, and reproduction in any medium, provided the original work is properly cited.

Peer-review history:

The peer review history for this paper can be accessed here: http://www.sciencedomain.org/review-history.php?iid=205\&id=12\&aid=1312 